\title{
Plasmon Mapping in Metallic Nanostructures and its Application to Single Molecule Surface Enhanced Raman Scattering: Imaging Electromagnetic Hot-Spots and Analyte Location
}

Final report for DOE Award number DE-SC0004792 (July 1, 2010 - June 1, 2013)

July 12, 2013

Principal Investigator:

Jon P. Camden, University of Tennessee, Department of Chemistry, Knoxville, TN 37996-1600

National Laboratory Partner: Oak Ridge National Lab, Oak Ridge, TN 37831

Beth S. Guiton and Stephen J. Pennycook, Materials Science and Technology Division

Unfunded Collaborators:

George C. Schatz, Department of Chemistry, Northwestern University, Evanston, IL 60208

David Masiello, Department of Chemistry, University of Washington, Seattle, WA 98195

\section{Introduction and Project Objectives}

The localized surface plasmon resonance (LSPR) results from the collective oscillation of the conduction band electrons in small $(d<<\lambda)$ metallic nanoparticles. The last several decades have seen an explosion in the study and application of plasmonic particles to problems ranging from fundamental optics\{Maier, 2001 \#177;Ozbay, 2006 \#174;Pendry, 2006 \#173;Smith, 2004 \#176\} to biology.\{Lyon, 1998 \#154;Kneipp, 1997 \#60;Kneipp, 2006 \#153\} Despite the fact that many applications of plasmonic particles rely on the large near-electric-field enhancements obtained when the particle is irradiated at the LSPR frequency it remains quite challenging to directly image these near-field enhancements. Theoretical predictions indicate that field enhancements can be confined to spaces as small as 1-2 $\mathrm{nm}$ which is well below the diffraction limit for optical microscopes. In contrast to optical microscopy, electron microscopy can easily image features on the nanometer scale, with the resolution of individual atoms being routinely achieved.\{Williams, $1996 \# 53\}$ Until recently, this feature of electron microscopy has not been applied to the imaging of low-energy (1-3eV) plasmon modes in nanoparticles.

When a material is exposed to an energetic beam of electrons, such as those in a transmission electron microscopy (TEM), some of these electrons will be inelastically scattered. The energy spectrum of the inelastically scattered electrons corresponds to excitations in the probed material. While electron energy loss spectroscopy (EELS) measurements in the low-loss region $(<50 \mathrm{eV})$ of processes such as bulk plasmons and Auger processes are well known,\{Williams, $1996 \# 53\}$ it is only in the last several years that useful information has begun to be extracted from the low energy end of this region, in which the LSPR modes lie ( $<3 \mathrm{eV},>400 \mathrm{~nm}$ ). This region of the spectrum is dominated by the zero-loss-peak (ZLP), formed by the elastically scattered electrons, which is many times more intense than the adjacent inelastically formed low-loss and core-loss edges. Deconvolution of the ZLP from the spectrum to resolve peaks in the very low LSPR region has recently become possible due to advances both in monochromation and the introduction of the cold field emission electron gun, both of which reduce the full width half maximum of the ZLP significantly.\{Williams, 1996 \#53\} 
In 2007 Nelayah et al.\{Nelayah, $2007 \# 31$ \} demonstrated the ability to measure spatially resolved EEL spectra with loss features very close to the zero loss peak in a scanning TEM (STEM). Using the combination of EELS and STEM they were able to map LSPR modes in individual Au nanoprisms down to $1.75 \mathrm{eV}(700 \mathrm{~nm})$. Bosman et al.,\{Bosman, 2007 \#57\} also in 2007, reported plasmon mapping studies of Ag and Au nanoparticles. Since these initial studies N'Gom et al. \{N'Gom, 2009 \#102;N'Gom, $2008 \# 103\}$ and Chu et al.\{Chu, $2009 \# 187\}$ have extended the initial studies to include coupled nanoparticles and explored dark plasmon modes, i.e. those that cannot be excited by far-field (plane wave) optical fields. These studies demonstrate the potential for EELS/STEM and energy filtered imaging TEM (EFTEM) to make a major contribution to our understanding of the near-field enhancements that drive current applications of plasmonic nanostructures. Despite the promise, fundamental details of the connection between EELS derived data and optical excitation remain unclear. \{Hohenester, 2009 \#42\} Therefore, a major component of this proposal is to elucidate the connection between optical and electron excitation of plasmon modes in metallic nanostructures.

\section{Accomplishments}

A. Developed a routine protocol for obtaining spatially resolved, low energy EELS spectra, and resonance Rayleigh scattering spectra from the same nanostructures.

In the first year of this proposal we have concentrated on the experimental methods and have studied several model systems. A survey of various nanoparticle geometries in both the optical and electron microscopes was made and the experimental parameters were optimized. Having solved several experimental challenges we can now routinely record the correlated optical/electron microscope measurements suggested in our proposal.

\section{B. Correlated optical scattering spectra and plasmon maps obtained using STEM/EELS.}

We obtained spatial maps of the localized surface plasmon modes of high aspect ratio silver nanorods using EELS and correlated them for the first time to optical data from the exact same particle. We applied multivariate statistical analysis (MVSA) to extract both light and dark plasmon modes, which show excellent agreement with classical electrodynamics calculations. Figure 1 reveals that vastly more information is available by utilizing EELS mapping. Where the optical spectrum measures only specific allowed transitions within a limited energy range, and the summed EEL spectrum shows overlapping collections of modes, MVSA reveals all of the symmetries and energies predicted by simulation - including both bright and dark modes, with a spatial resolution on the length scale of the plasmon itself. It is clear from comparison of the summed EEL spectra with the MVSA deconvoluted EELS plasmon maps, that, the relatively low energy resolution of the electron microscope leads to such great spectral overlap of modes that they cannot be identified without the deconvolution. Low EEL spectrum image (SI) mapping with MVSA, when combined with optical scattering data and electrodynamics modeling, as demonstrated in this report, provides an invaluable method for the characterization of plasmonic nanostructures whose complexity will only continue to increase in the future.

C. Imaged electromagnetic hot spots responsible for single-molecule surface-enhanced Raman scattering (SMSERS). 
We performed the first STEM/EELS imaging study of plasmonic nanostructures confirmed to be SMSERS-active. It has been widely accepted that the hot spots that give rise to SMSERS activity are located at the junction between two nanoparticles. By utilizing the bianalyte approach, \{Etchegoin, $2007 \# 380$;Le Ru, $2006 \# 158\}$ with correlated STEM/EELS, we demonstrated that electromagnetic hot spots can be excited with an electron beam when it is positioned at the periphery of a nanoparticle aggregate.
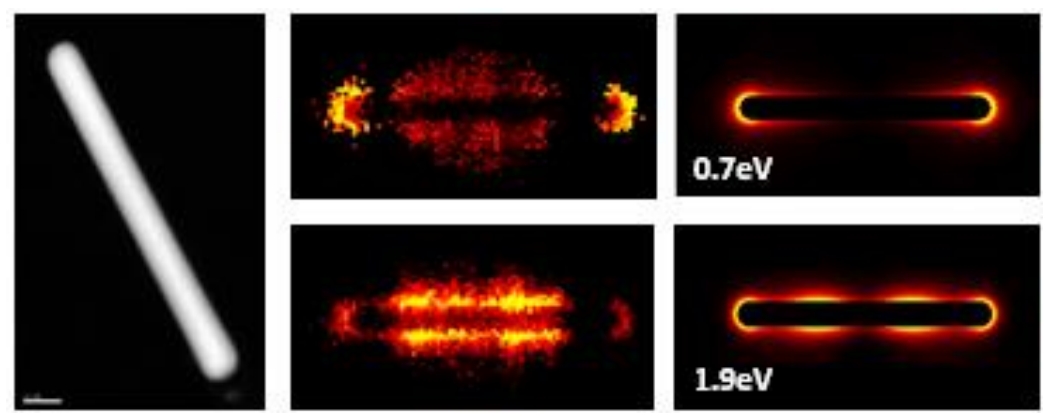

Figure 1: Annular dark field (ADF) image of a silver nanorod and plasmon maps showing the correlation between the experiment and theory of distinct plasmon modes at different energies.
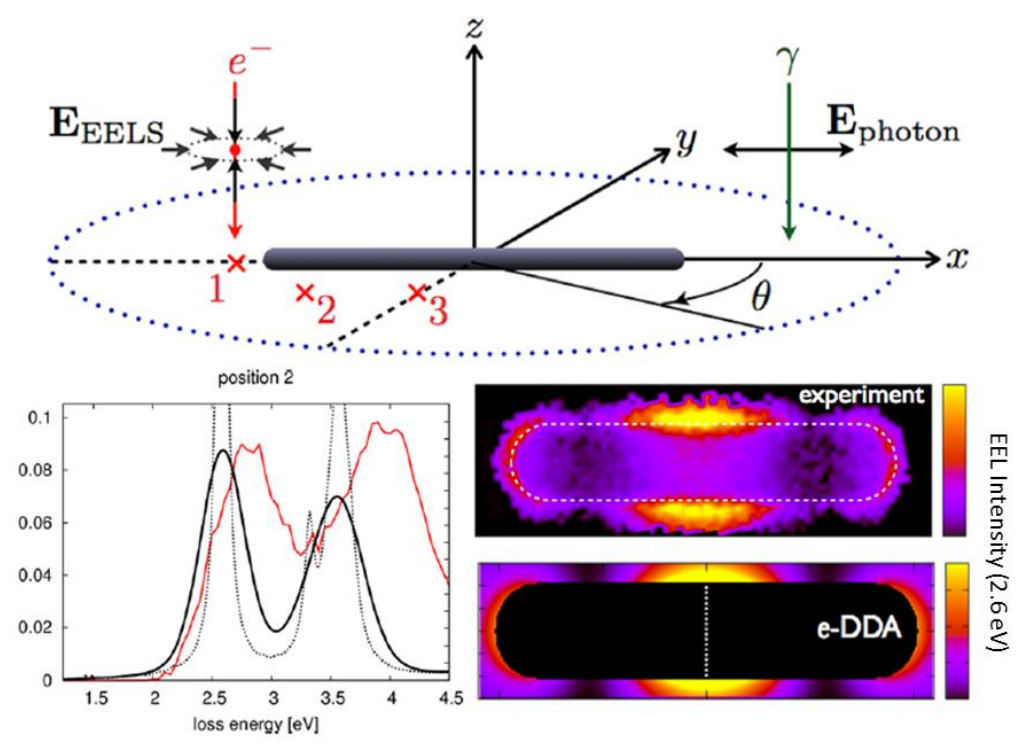

Figure 2: (Top) Schematic showing either fast-electron or photon driven excitations of a silver nanorod. Using our e-DDA simulations the EEL spectra can be computed for nanostructures of arbitrary shape and at any location external to the particle. (Bottom) Comparison between experiment (red) and theory (black) for point 2 (left) and for all locations at a loss energy of $2.6 \mathrm{eV}$ (right). The solid black line convolves the theory with a line width equal to the experimental resolution $(0.5 \mathrm{eV})$, otherwise no adjustable parameters are used. 


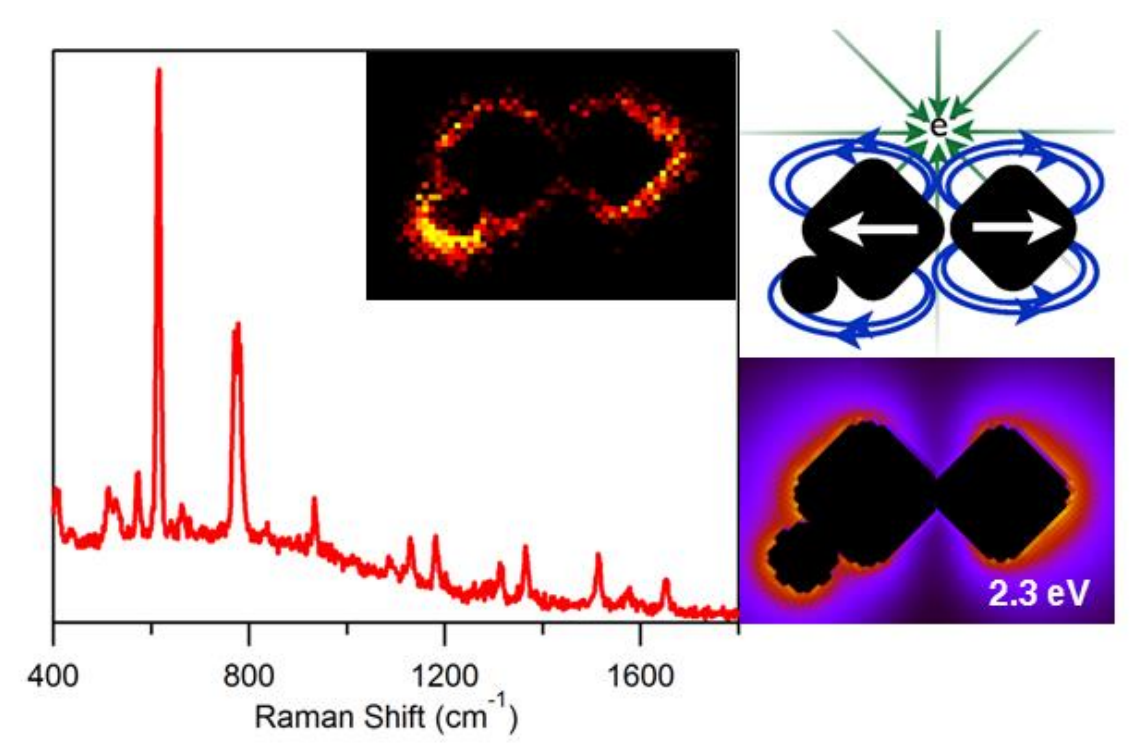

Figure 3: Single-molecule SERS spectrum for Rhodamine 6G with EELS characterization (experiment and theory). (L-R) Single-molecule SERS spectrum for Rhodamine 6G with EELS spatial map of nanostructure responsible for SMSERS activity (inset) and EELS theoretical simulation of the induced electric fields in the nanostructure by an electron beam.

\section{List of Publications}

Probing One-Photon Inaccessible Electronic States with High Sensitivity: Wavelength Scanned Surface Enhanced Hyper-Raman Scattering. C.B. Milojevich, D.W. Silverstein, L. Jensen, J.P. Camden, ChemPhysChem, 12, 101-103 (2011).

Spatial, Spectral, and Coherence Mapping of Single-molecule SERS Active Hot Spots. J.P. Litz, J.P. Camden, D.J. Masiello, J. Phys. Chem. Lett. 2, 1695-1700, (2011).

Correlated Optical Measurements and Plasmon Mapping of Silver Nanorods. B.S. Guiton, V. Iberi, S. Li, D.N. Leonard, C.M. Parish, P.G. Kotula, M. Varela, G.C. Schatz, S.J. Pennycook, J.P. Camden, Nano Letters, 11, 3482$3488,(2011)$.

Characterization of the Electron- and Photon-Driven Plasmonic Excitations of Metal nanorods. N.W. Bigelow, A. Vaschillo, V. Iberi, J.P. Camden, D.J. Masiello, ACS Nano, 6, 7497-7504, (2012).

Single Molecule Surface Enhanced Raman Scattering: Can STEM/EELS Image Electromagnetic Hot Spots? N. Mirsaleh-Kohan, V. Iberi, P.D. Simmons Jr., N.W. Bigelow, A. Vaschillo, M.M. Rowland, M. D. Best, S.J. Pennycook, D.J. Masiello, B.S. Guiton, J.P. Camden, J. Phys. Chem. Lett. 3, 2303-2309, (2012).

Directional Raman Scattering from Single Molecules in the Feed Gaps of Optical Antennas. D. Wang, W. Zhu, M.D. Best, J.P. Camden, K.B. Crozier, Nano Letters, 13, 2194-2198, (2013).

Understanding Plasmonic Properties in Metallic Nanostructures by Correlating Photonic and Electronic Excitations. V. Iberi, N. Mirsaleh-Kohan, J.P. Camden, J. Phys. Chem. Lett. 4, 1070-1078, (2013).

Signatures of Fano Interferences in the Electron Energy Loss Spectroscopy and Cathodoluminescence of SymmetryBroken Nanorod Dimers. N.W. Bigelow, A. Vaschillo, J.P. Camden, D.J. Masiello, ACS Nano, 7, 4511-4519, (2013). 


\section{Personnel}

Vighter Iberi, Graduate Student, 100\% effort

Philip Simmons, Graduate Student, 50\% effort

Nasrin Mirsaleh-Kohan, Postdoc, 100\% effort

Jon P. Camden, Principal Investigator, 3 months effort

\section{Current, Pending, and Planned Support}

The PI has support from the agencies listed below.

National Science Foundation, CHE-1150687; Period: 2012-2017; \$600,000 This project supports the development of surface enhanced nonlinear spectroscopy.

Department of Energy, DE-FOA-0000768; Period:2013-2016; $\$ 420,000$ This pending support continues the work initiated in the current project (DE-SC0004792)

\section{Cost Status}

Total Award Amount:

$\$ 450,000.00$

Total budgeted for Fiscal Year 2011: $\quad 420,000.00$

Total budgeted for Fiscal Year 2011: $\quad 15,000.00$

Total budgeted for Fiscal Year 2011: $\quad 15,000.00$

Actual to Date Expenditures:

$450,000.00$ 